\title{
INEQUALITIES FOR DIFFERENCES OF DYSON'S RANK FOR ALL ODD MODULI
}

\author{
Kathrin Bringmann and Ben Kane
}

\section{Introduction and Statement of results}

A partition of a non-negative integer $n$ is any non-increasing sequence of positive integers whose sum is $n$. As usual, let $p(n)$ denote the number of partitions of $n$. The partition function satisfies the famous "Ramanujan congruences" declaring that for $c \in\{5,7,11\}$ we have for all $n \geq 0$ that $p\left(c n+\delta_{c}\right) \equiv 0(\bmod c)$, where $\delta_{c}$ is defined by the congruence $24 \delta_{c} \equiv 1(\bmod c)$. In order to understand these from a combinatorial point of view, Dyson defined the rank of a partition as its largest part minus its number of parts [11]. Atkin and Swinnerton-Dyer [4] later proved that Dyson's rank indeed provides a combinatorial explanation of the congruences modulo 5 and 7 , but not the congruence modulo 11. To simplify notation, for integers $0 \leq a<c$, we let $N(a, c ; n)$ to be the number of partitions of $n$ whose rank is congruent to $a(\bmod c)$.

Rank differences have been the focus of several works and lead to interesting new automorphic forms, so called harmonic Maass forms. Harmonic Maass forms are generalizations of modular forms, in that they satisfy the same transformation law, and (weak) growth conditions at cusps, but instead of being holomorphic, they are annihilated by the weight $k$ hyperbolic Laplacian. As an example, consider the function

$$
f(q):=1+\sum_{n=1}^{\infty}(N(0,2, n)-N(1,2, n)) q^{n}=1+\sum_{n=1}^{\infty} \frac{q^{n^{2}}}{(1+q)^{2}\left(1+q^{2}\right)^{2} \cdots\left(1+q^{n}\right)^{2}},
$$

which is one of the third order mock theta functions Ramanujan defined in his last letter to Hardy [19]. Thanks to work of Zwegers [22] this function is now known to be the "holomorphic part" of a harmonic Maass form. A similar phenomenon is true for all the rank generating functions [7]. Asymptotic and exact formulas for the coefficients of $f(q)$ are proven by Dragonette [10], Andrews [1], and the first author and Ono [6]. These imply (as conjectured by Ramanujan) that

$$
\bar{\alpha}(n):=N(0,2, n)-N(1,2, n) \sim \frac{1}{2}(-1)^{n-1} n^{-\frac{1}{2}} \exp \left(\pi \sqrt{\frac{n}{6}-\frac{1}{144}}\right) .
$$

In particular we obtain that for $n$ sufficiently large (a statement which can be made precise) $\bar{\alpha}(n)$ is positive (resp. negative) if $n$ is odd (resp. even), which was first observed by Lewis [17] using combinatorial methods.

Received by the editors March 1, 2009

The first author was partially supported by NSF grant DMS-0757907. Part of the paper was written while the second author was in residence at IHES in France. He thanks the institute for providing a stimulating research environment. 
We next observe that the process of partition conjugation yields the identity

$$
N(a, c ; n)=N(c-a, c ; n) .
$$

In their proof of Dyson's rank conjecture Atkin and Swinnerton-Dyer [4] also showed some non-trivial identities including

$$
N(1,7 ; 7 n+1)=N(2,7 ; 7 n+1)=N(3,7 ; 7 n+1) .
$$

Moreover they related rank differences to infinite (modular) products, such as

$$
\sum_{n=0}^{\infty}(N(0,7 ; 7 n+6)-N(1,7 ; 7 n+6)) q^{n}=-\frac{\left(q ; q^{7}\right)_{\infty}^{2}\left(q^{6} ; q^{7}\right)_{\infty}^{2}\left(q^{7} ; q^{7}\right)_{\infty}^{2}}{(q ; q)_{\infty}} .
$$

With the benefit of retrospect, we may now view, thanks to Zwegers' thesis [21], such identities in the framework of automorphic forms occurring from relations between non-holomorphic parts of harmonic Maass forms. Their proof then boils down to a calculation of a finite number of Fourier coefficients. Using this idea the first author, Ono, and Rhoades [9] found infinite families of modular relations between rank differences which were then made explicit by S. Kang [15].

The situation is more complicated if one considers inequalities between rank differences since here a proof cannot be reduced to a finite computation of Fourier coefficients. For this reason only isolated examples of such inequalities have been shown so far. For example, Andrews and Lewis $[3,17]$ proved that

$$
\begin{aligned}
N(0,2 ; 2 n) & <N(1,2 ; 2 n) & & \text { if } n \geq 1, \\
N(0,4 ; n) & >N(2,4 ; n) & & \text { if } 26<n \equiv 0,1 \quad(\bmod 4), \\
N(0,4 ; n) & <N(2,4 ; n) & & \text { if } 26<n \equiv 2,3 \quad(\bmod 4) .
\end{aligned}
$$

Moreover, they conjectured (see Conjecture 1 of [3]).

Conjecture. (Andrews and Lewis)

For all $n>0$, we have

$$
\begin{array}{ll}
N(0,3 ; n)<N(1,3 ; n) & \text { if } n \equiv 0 \text { or } 2 \quad(\bmod 3), \\
N(0,3 ; n)>N(1,3 ; n) & \text { if } n \equiv 1 \quad(\bmod 3) .
\end{array}
$$

This conjecture was proven (up to a finite number of exceptions in which case one has equality) by the first author [5] using automorphic properties of the rank generating functions combined with the Circle Method. Here we obtain a similar result for the moduli 5,7 , and 9 . In view of (1.1) we only have to consider

$$
N(a, c, n)-N(b, c, n)
$$

with $0 \leq a<b \leq \frac{c-1}{2}$. The following theorem treats all the rank differences for moduli 5,7 , and 9 .

Theorem 1.1. For sufficiently large $n$ (see the Appendix for the exact statement) the following hold:

(1) We have

$$
N(a, 5,5 n+d)-N(b, 5,5 n+d)\left\{\begin{array}{cl}
<0 \quad \text { if }(a, b, d) \in\{(0, b, 0),(0,1,2),(a, 2,3)\} \\
>0 \quad \text { if }(a, b, d) \in\{(1,2,0),(0, b, 1),(1,2,2) \\
& (0,1,3)\}
\end{array}\right.
$$


(2) We have that the difference $N(a, 7,7 n+d)-N(b, 7,7 n+d)$ is

$$
\left\{\begin{array}{cc}
<0 \quad \text { if }(a, b, d) \in\{(0,1,2),(2,3,2),(1,2,3),(2,3,4),(0,1,6),(0,2,6),(1,2,6)\}, \\
>0 \quad \text { if }(a, b, d) \in\{(0, b, 0),(1, b, 0),(0, b, 1),(0,2,2),(1, b, 2),(0,1,3), \\
& (0,3,3),(2,3,3),(0,2,4),(1,2,4),(0,3,6),(1,3,6),(2,3,6)\} .
\end{array}\right.
$$

(3) We have that the difference $N(a, 9,3 n+d)-N(b, 9,3 n+d)$ is

$$
\left\{\begin{aligned}
<0 & \text { if }(a, b, d) \in\{(0,1,0),(1,3,1),(2,3,1),(0,1,2),(0,2,2),(3,4,2)\}, \\
>0 & \text { if }(a, b, d) \in\{(0,2,0),(0,3,0),(0,4,0),(1, b, 0),(2, b, 0),(0, b, 1), \\
& (1,2,1),(a, 4,1),(0,3,2),(1, b, 2),(2, b, 2)\} .
\end{aligned}\right.
$$

Remarks.

1) Some comments are in order concerning Theorem 1.1. Of the above inequalities for the moduli 5 and 7 some were conjectured and some were proven by Garvan $[12,13]$ using combinatorial methods. For completeness we decided to give a full list for all (odd) moduli here (the remaining cases can be found in Theorem 1.2). We further recall that the above mentioned equalities (1.2) and (1.3) arose from relations between non-holomorphic parts and thus from a modern point of view can be seen as statements about modular forms. Similarly, some of the inequalities in Theorem 1.1, in particular those shown by Garvan, are statements about the positivity of Fourier coefficients of modular forms. Of particular interest are those inequalities for which this is not the case, i.e., the associated harmonic Maass forms also have nontrivial nonholomorphic parts, for example the case $(0,1,0)$ (for moduli 5 ), which is related to the mock theta conjectures going back to Ramanujan. These are a list of ten identities involving Ramanujan's mock theta functions of order 5. Andrews and Garvan [2] proved that these are equivalent to the truth of the following pair of combinatorial identities

$$
\begin{aligned}
N(1,5,5 n) & =N(0,5,5 n)+\rho_{0}(n), \\
2 N(2,5,5 n+3) & =N(1,5,5 n+3)+N(0,5,5 n+3)+\rho_{1}(n)+1,
\end{aligned}
$$

where $\rho_{0}(n)$ is the number of partitions of $n$ with unique smallest part and all other parts $\leq$ the double of the smallest part and $\rho_{1}(n)$ is the number of partitions of $n$ with unique smallest part and all other parts $\leq$ one plus the double of the smallest part. Clearly these statistics are non-negative so the first mock theta identity implies the case $(0,1,0)$ of Theorem 1.1. The mock theta conjectures remained open until Hickerson's important paper [14] in which he used lengthy and highly complicated combinatorial methods.

2) From [4], we know that in the missing cases equality holds for the cases 5 and 7 , while Santa-Gadea [20] has shown that equality holds in the two missing cases for $c=9$.

3) The much weaker statement that both the greater than and less than inequalities in (1), (2), (3) must be satisfied for at least one choice of $n$ and $d$ follows from an observation of Knopp, Kohnen, and Pribitkin [16, p. 274].

Given the nature of the Andrews-Lewis Conjecture and Theorem 1.1 one might expect a similar behavior in the case for general moduli, namely that rank inequalities are dictated by congruence conditions. However, we show that in the case of higher moduli the rank inequality is surprisingly unaffected by the residue class. 
Theorem 1.2. Assume that $c>9$ is an odd integer. Then for $0 \leq a<b \leq \frac{c-1}{2}$ we have for $n>N_{a, b, c}$, where $N_{a, b, c}$ is an explicit constant, the inequality

$$
N(a, c, n)>N(b, c, n) .
$$

Remarks.

1) The proof of Theorem 1.2 gives an explicit algorithm to determine the bound $N_{a, b, c}$. Using this algorithm we obtained the bounds necessary to show Theorem 1.1. The bounds employed in the proof of Theorem 1.2 can be used to obtain the bounds necessary to show Theorem 1.1. The only difference occurs in determining the main term. Since the proof merely amounts to a numerical calculation we chose to not include further details in the paper.

2) We recall that Atkin and Swinnerton-Dyer's proof of Dyson's rank conjecture relied on identities like (1.2). Theorem 1.2 shows that although there are infinitely many identities of type (1.3) by [9], identities of type (1.2) cannot exist for moduli above 9. Thus the rank cannot be used to dissect the partition function for any of these moduli (not even for sufficiently large $n$ ).

\section{Proof of Theorem 1.2}

For simplicity we throughout assume that $c$ is a prime, since the case of composite $c$ is treated similarly but with slightly varying bounds. We write

$$
\sum_{n}(N(a, c ; n)-N(b, c ; n)) q^{n}=\frac{2}{c} \sum_{j=1}^{\frac{c-1}{2}} \rho_{j}(a, b, c) R\left(\zeta_{c}^{j} ; q\right),
$$

where $\zeta_{c}:=e^{\frac{2 \pi i}{c}}$ and

$$
R(z ; q):=\sum_{n=0}^{\infty} \sum_{m \in \mathbb{Z}} N(n, m) z^{m} q^{n},
$$

with $N(m, n)$ counting the number of partitions of $n$ with rank $m$. Moreover,

$$
\rho_{j}(a, b, c):=\left(\cos \left(\frac{2 \pi a j}{c}\right)-\cos \left(\frac{2 \pi b j}{c}\right)\right) .
$$

Define the coefficients $A\left(\frac{j}{c} ; n\right)$ by

$$
R\left(\zeta_{c}^{j} ; q\right)=: 1+\sum_{n=1}^{\infty} A\left(\frac{j}{c} ; n\right) q^{n} .
$$

To determine the asymptotic behavior of (2.1), we use Theorem 1.1 of [5]. To state this, we require some notation. We let $k$ and $h$ be coprime integers, and define $h^{\prime}$ by $h h^{\prime} \equiv-1(\bmod k)$ if $k$ is odd and by $h h^{\prime} \equiv-1(\bmod 2 k)$ if $k$ is even. Moreover, if $c \nmid k$, we define $0<l<c$ by the congruence $l \equiv j k(\bmod c)$. Moreover, if $\frac{f}{c} \in(0,1) \backslash\left\{\frac{1}{2}, \frac{1}{6}, \frac{5}{6}\right\}$, then define the integer $s(f, c)$ by

$$
s(f, c):= \begin{cases}0 & \text { if } 0<\frac{f}{c}<\frac{1}{6}, \\ 1 & \text { if } \frac{1}{6}<\frac{f}{c}<\frac{1}{2} \\ 2 & \text { if } \frac{1}{2}<\frac{f}{c}<\frac{5}{6} \\ 3 & \text { if } \frac{5}{6}<\frac{f}{c}<1\end{cases}
$$


In particular, set $s:=s(l, c)$. Let $\omega_{h, k}$ be the multiplier occurring in the transformation law of the partition function $p(n)$ (see [18]) which can be written in terms of Gauss sums and in particular satisfies $\left|\omega_{h, k}\right|=1$. Moreover we define, for $n, m \in \mathbb{Z}$, the following sums of Kloosterman type

$$
B_{j, c, k}(n, m):=(-1)^{j k+1} \sin \left(\frac{\pi j}{c}\right) \sum_{h} \frac{\omega_{h, k}}{(\bmod k)^{*}} \cdot e^{-\frac{3 \pi i j^{2} h^{\prime}}{c}} \cdot e^{\frac{2 \pi i}{k}\left(n h+m h^{\prime}\right)}
$$

if $c \mid k$, and

$$
D_{j, c, k}(n, m):=(-1)^{j k+l} \sum_{h} \omega_{h, k} \cdot e^{\frac{2 \pi i}{k}\left(n h+m h^{\prime}\right)} .
$$

Here the sums run through all primitive residue classes modulo $k$. Moreover, for $c \nmid k$, let

$$
\delta_{j, c, k, r}:= \begin{cases}-\left(\frac{1}{2}+r\right) \frac{l}{c}+\frac{3}{2}\left(\frac{l}{c}\right)^{2}+\frac{1}{24} & \text { if } 0<\frac{l}{c}<\frac{1}{6} \\ -\frac{5 l}{2 c}+\frac{3}{2}\left(\frac{l}{c}\right)^{2}+\frac{25}{24}-r\left(1-\frac{l}{c}\right) & \text { if } \frac{5}{6}<\frac{l}{c}<1 \\ 0 & \text { otherwise }\end{cases}
$$

and for $0<\frac{l}{c}<\frac{1}{6}$ or $\frac{5}{6}<\frac{l}{c}<1$

$$
m_{j, c, k, r}:= \begin{cases}\frac{1}{2 c^{2}}\left(-3 j^{2} k^{2}+6 l j k-j k c-3 l^{2}+l c-2 j r k c+2 l c r\right) & \text { if } 0<\frac{l}{c}<\frac{1}{6}, \\ \frac{1}{2 c_{1}^{2}}\left(-6 j k c-3 j^{2} k^{2}+6 l j k+j k c+6 l c\right. & \text { if } \frac{5}{6}<\frac{l}{c}<1 . \\ \left.-3 l^{2}-2 c^{2}-l c+2 j r k c+2 c(c-l) r\right) & \end{cases}
$$

In [5], the following asymptotic formulas for the coefficients $A\left(\frac{j}{c} ; n\right)$ were shown using the Circle Method.

Theorem 2.1. If $0<j<c$ are coprime integers and $c$ is odd, then for positive integers $n$ we have that

$$
\begin{aligned}
A\left(\frac{j}{c} ; n\right) & =\frac{4 \sqrt{3} i}{\sqrt{24 n-1}} \sum_{\substack{1 \leq k \leq \sqrt{n} \\
c \mid k}} \frac{B_{j, c, k}(-n, 0)}{\sqrt{k}} \sinh \left(\frac{\pi \sqrt{24 n-1}}{6 k}\right)+\frac{8 \sqrt{3} \cdot \sin \left(\frac{\pi j}{c}\right)}{\sqrt{24 n-1}} \\
& \times \sum_{\substack{1 \leq k \leq \sqrt{n} \\
\text { c.k } \\
r \geq 0 \\
\delta_{j, c, k}>r>0}} \frac{D_{j, c, k}\left(-n, m_{j, c, k, r}\right)}{\sqrt{k}} \sinh \left(\frac{\pi \sqrt{2 \delta_{j, c, k, r}(24 n-1)}}{\sqrt{3} k}\right)+O_{c}\left(n^{\epsilon}\right) .
\end{aligned}
$$

\section{Remarks.}

We note that by work of the first author and Ono [8] an exact formula for $A\left(\frac{j}{c} ; n\right)$ involving infinite sums on $k$ is known. To prove Theorem 1.2 one could also employ this formula and bound the tails (i.e., those terms with large $k$ ) for example by using spectral theory. However it seems much more complicated to make these bounds explicit.

Inserting Theorem 2.1 into (2.1) yields that

$$
N(a, c ; n)-N(b, c ; n)=\sum_{j=1}^{\frac{c-1}{2}}\left(S_{j}(a, b ; c)+T_{j}(a, b ; c)\right)+O_{c}\left(n^{\epsilon}\right),
$$


where

$$
\begin{aligned}
S_{j}(a, b ; c):= & \rho_{j}(a, b, c) \frac{8 \sqrt{3} i}{c \sqrt{24 n-1}} \sum_{\substack{1 \leq k \leq \sqrt{n} \\
c \mid k}} \frac{B_{j, c, k}(-n, 0)}{\sqrt{k}} \sinh \left(\frac{\pi \sqrt{24 n-1}}{6 k}\right), \\
T_{j}(a, b ; c):= & \rho_{j}(a, b, c) \frac{16 \sqrt{3} \cdot \sin \left(\frac{\pi j}{c}\right)}{c \sqrt{24 n-1}} \\
& \times \sum_{\substack{1 \leq k \leq \sqrt{n} \\
c k+\\
r \geq 0 \\
\delta_{j, c, k, r}>0}} \frac{D_{j, c, k}\left(-n, m_{j, c, k, r}\right)}{\sqrt{k}} \sinh \left(\frac{\pi \sqrt{2 \delta_{j, c, k, r}(24 n-1)}}{\sqrt{3} k}\right) .
\end{aligned}
$$

In order to show Theorem 1.2, we must determine which terms give the main contribution to (2.3) and then bound the other terms explicitly.

2.1. Determining the main terms. We first compare the occurring arguments of the hyperbolic sines in (2.4) and (2.5) in order to determine the main terms. In the sums $S_{j}$, one can directly see that the largest argument occurs when $k=c$. In $T_{j}$ the term coming from $k=1, r=0$, and $j=1$ has the argument

$$
\frac{\pi \sqrt{2 \delta_{0}(24 n-1)}}{\sqrt{3}},
$$

where $\delta_{0}:=\frac{3}{2 c^{2}}-\frac{1}{2 c}+\frac{1}{24}$. We will proceed to show that this gives the main contribution to (2.3). Using that $c>7$, it is not hard to see that the argument in the hyperbolic sine is smaller in the terms coming from $S_{j}$. Turning to $T_{j}$, we may assume that $r=0$, since for fixed $j$ and $k$ this yields the largest argument. Assuming without loss of generality that $0<\frac{l}{c}<\frac{1}{6}$, we have

$$
\delta_{j, c, k, 0}=-\frac{l}{2 c}+\frac{3}{2}\left(\frac{l}{c}\right)^{2}+\frac{1}{24} \leq \delta_{0} .
$$

Moreover, for $k=1$, we have $l=j$ and $\delta_{j, c, 1,0}<\delta_{0}$, when $j \neq 1$, which yields that the main contribution occurs for $k=1, r=0$, and $j=1$.

The main term coming from $k=1, r=0$, and $j=1$ gives the contribution

$$
T_{1}(a, b ; c)=\frac{2}{c} \rho_{1}(a, b, c) \frac{8 \sqrt{3} \sin \left(\frac{\pi}{c}\right)}{\sqrt{24 n-1}} \sinh \left(\frac{\pi \sqrt{2 \delta_{0}(24 n-1)}}{\sqrt{3}}\right) .
$$

We note that the sign of this equation is entirely determined by the sign of $\rho_{j}(a, b, c)$, which is clearly positive since $0 \leq a<b \leq \frac{c-1}{2}$. This yields that $N(a, c, n)>N(b, c, n)$ for $n$ sufficiently large. In the following we will make this statement more precise.

2.1.1. Bounding the contribution of $S_{j}$ and $T_{j}$. We first consider $S_{j}$ and estimate 


$$
\begin{aligned}
& \left|S_{j}(a, b ; c)\right| \\
& \leq \frac{8\left|\rho_{j}(a, b, c)\right| \sqrt{3}}{c \sqrt{24 n-1}} \sum_{\substack{1 \leq k \leq \sqrt{n} \\
c \mid k}} \frac{\left|B_{j, c, k}(-n, 0)\right|}{\sqrt{k}} \sinh \left(\frac{\pi \sqrt{24 n-1}}{6 k}\right) \\
& \leq \frac{8\left|\rho_{j}(a, b, c)\right| \sqrt{3}}{c \sqrt{24 n-1}}\left|\sin \left(\frac{\pi j}{c}\right)\right| \sinh \left(\frac{\pi \sqrt{24 n-1}}{6 c}\right) \sum_{\substack{1 \leq k \leq \sqrt{n} \\
c \mid k}} k^{-\frac{1}{2}} \sum_{\substack{h=1 \\
(h, k)=1}}^{k} \frac{1}{\left|\sin \left(\frac{\pi h}{c}\right)\right|} .
\end{aligned}
$$

We estimate the inner sum using the inequality

$$
\sum_{\substack{h=1 \\(h, k)=1}}^{k} \frac{1}{\left|\sin \left(\frac{\pi h}{c}\right)\right|} \leq \frac{2 k}{c} \sum_{h=1}^{\frac{c-1}{2}} \frac{1}{\left|\sin \left(\frac{\pi h}{c}\right)\right|} \leq \frac{2 k}{\pi} \sum_{h=1}^{\frac{c-1}{2}} \frac{1}{h\left(1-\frac{\pi^{2}}{24}\right)} \leq \frac{2 k\left(1+\log \left(\frac{c-1}{2}\right)\right)}{\pi\left(1-\frac{\pi^{2}}{24}\right)} .
$$

This yields

$$
\begin{aligned}
& \left|S_{j}(a, b ; c)\right| \\
& \quad \leq \frac{16\left|\rho_{j}(a, b, c)\right| \sqrt{3}}{c \sqrt{24 n-1}} \frac{\left|\sin \left(\frac{\pi j}{c}\right)\right|\left(1+\log \left(\frac{c-1}{2}\right)\right)}{\pi\left(1-\frac{\pi^{2}}{24}\right)} \sinh \left(\frac{\pi \sqrt{24 n-1}}{6 c}\right) \sum_{\substack{1 \leq k \leq \sqrt{n} \\
c \mid k}} k^{\frac{1}{2}} \\
& \leq \frac{64\left(1+\log \left(\frac{c-1}{2}\right)\right) n^{\frac{3}{4}}}{\sqrt{24 n-1} c^{2} \sqrt{3} \pi\left(1-\frac{\pi^{2}}{24}\right)} \sinh \left(\frac{\pi \sqrt{24 n-1}}{6 c}\right) .
\end{aligned}
$$

We note that this estimate could be improved in specific cases.

We next explicitly estimate the error coming from $T_{j}$ and we will trivially bound $D_{j, c, k}\left(-n, m_{j, c, k, r}\right)$ by $k$ in all instances. Using the above, we see that every term inside the sum with $k \geq 2$, for each $r$ such that $\delta_{j, c, k, r}>0$, can be bounded against

$$
k^{\frac{1}{2}} \sinh \left(\pi \frac{\sqrt{2 \delta_{0}(24 n-1)}}{2 \sqrt{3}}\right) \leq \frac{k^{\frac{1}{2}}}{2} e^{\pi \frac{\sqrt{2 \delta_{0}(24 n-1)}}{2 \sqrt{3}}} .
$$

Due to symmetry we may in the following assume that $\frac{j}{c}<\frac{1}{6}$. We note that the number of $r$ satisfying $\delta_{j, c, k, r}>0$ is decreasing as a function of $l$ and thus has its maximum at $l=1$, in which case it equals

$$
\left\lfloor\frac{c}{24}+\frac{1}{2}+\frac{3}{2 c}\right\rfloor<\frac{c+18}{24} .
$$

Thus the contribution coming from $k \neq 1$ can be estimated against

$$
\frac{4(c+18)}{3 \sqrt{3} c \sqrt{24 n-1}} n^{\frac{3}{4}} e^{\pi \frac{\sqrt{2 \delta_{0}(24 n-1)}}{2 \sqrt{3}}} .
$$

Moreover, letting $\delta_{1}:=\delta_{2, c, 1,0}$, the contribution of $k=1$ can be estimated against

$$
\frac{2(c+18)}{\sqrt{3} c \sqrt{24 n-1}} e^{\pi \frac{\sqrt{2 \delta_{1}(24 n-1)}}{\sqrt{3}}} .
$$


2.2. Estimation of the error term arising in the Circle Method. We next explicitly estimate the error terms which occurred in Theorem 2.1 from using the Circle Method. For the readers convenience, we first recall the required set up of the Circle Method following [5].

2.2.1. Set up. By Cauchy's Theorem we have for $n>0$

$$
A\left(\frac{j}{c} ; n\right)=\frac{1}{2 \pi i} \int_{C} \frac{N\left(\frac{j}{c} ; q\right)}{q^{n+1}} d q
$$

where

$$
N\left(\frac{j}{c} ; q\right):=R\left(\zeta_{c}^{j} ; q\right)
$$

and where $C$ is an arbitrary path inside the unit circle surrounding 0 counterclockwise. Choosing the circle with radius $e^{-\frac{2 \pi}{n}}$ and as a parametrisation $q=e^{-\frac{2 \pi}{n}+2 \pi i t}$ with $0 \leq t \leq 1$, gives

$$
A\left(\frac{j}{c} ; n\right)=\int_{0}^{1} N\left(\frac{j}{c} ; e^{-\frac{2 \pi}{n}+2 \pi i t}\right) \cdot e^{2 \pi-2 \pi i n t} d t
$$

Define

$$
\vartheta_{h, k}^{\prime}:=\frac{1}{k\left(k_{1}+k\right)}, \quad \vartheta_{h, k}^{\prime \prime}:=\frac{1}{k\left(k_{2}+k\right)},
$$

where $\frac{h_{1}}{k_{1}}<\frac{h}{k}<\frac{h_{2}}{k_{2}}$ are adjacent Farey fractions in the Farey sequence of order $N:=\left\lfloor n^{1 / 2}\right\rfloor$. From the theory of Farey fractions it is known that

$$
\frac{1}{k+k_{j}} \leq \frac{1}{N+1} \quad(j=1,2)
$$

We decompose the path of integration in paths along the Farey arcs $-\vartheta_{h, k}^{\prime} \leq \Phi \leq \vartheta_{h, k}^{\prime \prime}$, where $\Phi=t-\frac{h}{k}$ and $0 \leq h \leq k \leq N$ with $(h, k)=1$. Thus

$$
A\left(\frac{j}{c} ; n\right)=\sum_{h, k} e^{-\frac{2 \pi i h n}{k}} \int_{-\vartheta_{h, k}^{\prime}}^{\vartheta_{h, k}^{\prime \prime}} N\left(\frac{j}{c} ; e^{\frac{2 \pi i}{k}(h+i z)}\right) \cdot e^{\frac{2 \pi n z}{k}} d \Phi,
$$

where $z=\frac{k}{n}-k \Phi i$. Applying the transformation law for the rank generating functions shown in [5] gives

$$
A\left(\frac{j}{c} ; n\right)=\sum_{1}+\sum_{2}+\sum_{3}
$$


where

$$
\begin{aligned}
\sum_{1}:= & i \sin \left(\frac{\pi j}{c}\right) \sum_{\substack{h, k \\
c \mid k}} \omega_{h, k} \frac{(-1)^{j k+1}}{\sin \left(\frac{\pi j h^{\prime}}{c}\right)} \cdot e^{-\frac{3 \pi i j^{2} k h^{\prime}}{c^{2}}-\frac{2 \pi i h n}{k}} \\
& \times \int_{-\vartheta_{h, k}^{\prime}}^{\vartheta_{h, k}^{\prime \prime}} z^{-\frac{1}{2}} \cdot e^{\frac{2 \pi z}{k}\left(n-\frac{1}{24}\right)+\frac{\pi}{12 k z}} N\left(\frac{j h^{\prime}}{c} ; q_{1}\right) d \Phi, \\
\sum_{2}:= & -4 i \sin \left(\frac{\pi j}{c}\right) \sum_{h, k} \omega_{h, k}(-1)^{j k+l} e^{-\frac{2 \pi i h^{\prime} s j}{c}-\frac{3 \pi i h^{\prime} j^{2} k}{c^{2}}+\frac{6 \pi i h^{\prime} l j}{c^{2}}-\frac{2 \pi i h n}{k}} \\
& \times \int_{-\vartheta_{h, k}^{\prime}}^{\vartheta_{h, k}^{\prime \prime}} z^{-\frac{1}{2}} \cdot e^{\frac{2 \pi z}{k}\left(n-\frac{1}{24}\right)+\frac{\pi}{12 k z} \cdot q_{1}^{\frac{s l}{c}-\frac{3 l^{2}}{2 c^{2}}} \cdot N\left(j h^{\prime}, l, c ; q_{1}\right) d \Phi,} \\
\sum_{3}:= & \sin ^{2}\left(\frac{\pi j}{c}\right) \sum_{h, k} \frac{\omega_{h, k}}{k} \cdot e^{-\frac{2 \pi i h n}{k}} \sum_{(\bmod k)}(-1)^{\nu} e^{-\frac{3 \pi i h^{\prime} \nu^{2}}{k}+\frac{\pi i h^{\prime} \nu}{k}} \\
& \times \int_{-\vartheta_{h, k}^{\prime}}^{\vartheta_{h, k}^{\prime \prime}} e^{\frac{2 \pi z}{k}\left(n-\frac{1}{24}\right) \cdot z^{\frac{1}{2}} \cdot I_{j, c, k, \nu}(z) d \Phi .}
\end{aligned}
$$

Here $q_{1}:=e^{\frac{2 \pi}{k}\left(h^{\prime}+\frac{i}{z}\right)}$,

$$
\begin{array}{r}
N(a, b, c ; q):=\frac{i}{2(q ; q)_{\infty}}\left(\sum_{m=0}^{\infty} \frac{(-1)^{m} e^{-\frac{\pi i a}{c}} \cdot q^{\frac{m}{2}(3 m+1)+m s(b, c)+\frac{b}{2 c}}}{1-e^{-\frac{2 \pi i a}{c}} \cdot q^{m+\frac{b}{c}}}\right. \\
\left.-\sum_{m=1}^{\infty} \frac{(-1)^{m} e^{\frac{\pi i a}{c}} \cdot q^{\frac{m}{2}(3 m+1)-m s(b, c)-\frac{b}{2 c}}}{1-e^{\frac{2 \pi i a}{c}} \cdot q^{m-\frac{b}{c}}}\right),
\end{array}
$$

and

$$
I_{j, c, k, \nu}(z):=\int_{\mathbb{R}} e^{-\frac{3 \pi z x^{2}}{k}} \cdot H_{j, c}\left(\frac{\pi i \nu}{k}-\frac{\pi i}{6 k}-\frac{\pi z x}{k}\right) d x
$$

with

$$
H_{j, c}(x):=\frac{\cosh (x)}{\sinh \left(x+\frac{\pi i j}{c}\right) \cdot \sinh \left(x-\frac{\pi i j}{c}\right)} .
$$

To estimate $\sum_{1}$, we split $N\left(\frac{h j^{\prime}}{c} ; q_{1}\right)$ into a contribution coming from the constant term (which will be part of the main contribution) and an error contribution coming from the remaining terms. We denote the associated sums by $S_{1}$ and $S_{2}$. Throughout we need the easily verified fact that $\operatorname{Re}(z)=\frac{k}{n}, \operatorname{Re}\left(\frac{1}{z}\right)>\frac{k}{2},|z|^{-\frac{1}{2}} \leq n^{\frac{1}{2}} \cdot k^{-\frac{1}{2}}$, and $\vartheta_{h, k}^{\prime}+\vartheta_{h, k}^{\prime \prime} \leq \frac{2}{k(N+1)}$.

2.2.2. Estimation of $S_{2}$. We get

$$
S_{2} \leq 2\left|\sin \left(\frac{\pi j}{c}\right)\right| e^{2 \pi} \sum_{\substack{k \leq N \\ c \mid k}} k^{-\frac{3}{2}} \sum_{\substack{h=1 \\(h, k)=1}}^{k-1} \frac{1}{\left|\sin \left(\frac{\pi h}{c}\right)\right|} \max _{z}\left|e^{\frac{\pi}{12 k z}}\left(N\left(\frac{h}{c} ; q_{1}\right)-1\right)\right| .
$$


To estimate $\left|e^{\frac{\pi}{12 k z}}\left(N\left(\frac{h}{c} ; q_{1}\right)-1\right)\right|$, recall that

$$
\begin{aligned}
N\left(\frac{h}{c} ; q_{1}\right)=\frac{1}{\left(q_{1} ; q_{1}\right)_{\infty}}+\frac{\left(1-\zeta_{c}^{h}\right)}{\left(q_{1} ; q_{1}\right)_{\infty}} \sum_{m=1}^{\infty} \frac{(-1)^{m} q_{1}^{\frac{m}{2}(3 m+1)}}{1-\zeta_{c}^{h} q_{1}^{m}} & \quad+\frac{\left(1-\zeta_{c}^{-h}\right)}{\left(q_{1} ; q_{1}\right)_{\infty}} \sum_{m=1}^{\infty} \frac{(-1)^{m} q_{1}^{\frac{m}{2}(3 m+1)}}{1-\zeta_{c}^{-h} q_{1}^{m}}
\end{aligned}
$$

Thus $\left|e^{\frac{\pi}{12 k z}}\left(N\left(\frac{h}{c} ; q_{1}\right)-1\right)\right|$ can be bounded against

$$
\begin{aligned}
& e^{\frac{\pi}{24}} \sum_{m=1}^{\infty} p(m) e^{-\pi m}+e^{\frac{\pi}{24}} \sum_{m=0}^{\infty} p(m) e^{-\pi m} \sum_{m=1}^{\infty} e^{-\frac{\pi m(3 m+1)}{2}}\left|\frac{1-\zeta_{c}^{h}}{1-\zeta_{c}^{h} q_{1}^{m}}+\frac{1-\zeta_{c}^{-h}}{1-\zeta_{c}^{-h} q_{1}^{m}}\right| \\
& \leq e^{\frac{\pi}{24}} \sum_{m=1}^{\infty} p(m) e^{-\pi m}+2\left(1+\left|\cos \left(\frac{\pi}{c}\right)\right|\right) e^{\frac{\pi}{24}} \sum_{m=0}^{\infty} p(m) e^{-\pi m} \sum_{m=1}^{\infty} \frac{e^{-\frac{\pi m(3 m+1)}{2}}}{1-e^{-\pi m}}
\end{aligned}
$$

This gives the estimate

$$
S_{2} \leq 2 e^{2 \pi}\left|\sin \left(\frac{\pi j}{c}\right)\right| e^{\frac{\pi}{24}}\left(c_{2}+2\left(1+\left|\cos \left(\frac{\pi}{c}\right)\right|\right) c_{1}\left(1+c_{2}\right)\right) \sum_{\substack{k \leq N \\ c \mid k}} k^{-\frac{3}{2}} \sum_{h=1}^{k-1} \frac{1}{\left|\sin \left(\frac{\pi h}{c}\right)\right|},
$$

where

$$
c_{1}:=\sum_{m=1}^{\infty} \frac{e^{-\frac{\pi m(3 m+1)}{2}}}{1-e^{-\pi m}}, \quad c_{2}:=\sum_{m=1}^{\infty} p(m) e^{-\pi m} .
$$

Using (2.7) and estimating the sum on $k$ yields

$$
S_{2} \leq \frac{8 e^{2 \pi+\frac{\pi}{24}}\left(c_{2}+2\left(1+\left|\cos \left(\frac{\pi}{c}\right)\right|\right) c_{1}\left(1+c_{2}\right)\right)\left|\sin \left(\frac{\pi j}{c}\right)\right| n^{\frac{1}{4}}\left(1+\log \left(\frac{c-1}{2}\right)\right)}{\pi\left(1-\frac{\pi^{2}}{24}\right) c}
$$

2.2.3. Terms in $\sum_{2}$ with positive exponent. We next estimate the terms in $\sum_{2}$ corresponding to positive exponents in the $q_{1}$-expansion of $e^{\frac{\pi}{12 k z}} q_{1}^{\frac{s l}{c}-\frac{3 l^{2}}{2 c^{2}}} N\left(j h^{\prime}, l, c ; q_{1}\right)$ which will contribute to the error. We denote this series by $N^{*}\left(j h^{\prime}, l, c ; q_{1}\right)$ and denote the whole associated sum by $T_{2}$. We have

$$
T_{2} \leq 8 e^{2 \pi}\left|\sin \left(\frac{\pi j}{c}\right)\right| \sum_{\substack{h, k \\ c \nmid k}} k^{-\frac{3}{2}} \max _{z}\left|N^{*}\left(h, l, c ; q_{1}\right)\right|
$$

To estimate $N^{*}$, we write

$$
\begin{aligned}
& N\left(h, l, c ; q_{1}\right)=-\frac{i \zeta_{2 c}^{h} q_{1}^{-\frac{l}{2 c}}}{2\left(q_{1} ; q_{1}\right)_{\infty}\left(1-\zeta_{c}^{h} q_{1}^{-\frac{l}{c}}\right)}+\frac{i \zeta_{2 c}^{h} q_{1}^{-\frac{l}{2 c}+2-s}}{2\left(q_{1} ; q_{1}\right)_{\infty}\left(1-\zeta_{c}^{h} q_{1}^{1-\frac{l}{c}}\right)} \\
& \quad-\frac{i \zeta_{2 c}^{h} q_{1}^{-\frac{l}{2 c}}}{2\left(q_{1} ; q_{1}\right)_{\infty}} \sum_{m=2}^{\infty} \frac{(-1)^{m} q_{1}^{\frac{m}{2}(3 m+1)-s m}}{1-\zeta_{c}^{h} q_{1}^{m-\frac{l}{c}}}+\frac{i \zeta_{2 c}^{-h} q_{1}^{\frac{l}{2 c}}}{2\left(q_{1} ; q_{1}\right)_{\infty}} \sum_{m=1}^{\infty} \frac{(-1)^{m} q_{1}^{\frac{m}{2}(3 m+1)+s m}}{1-\zeta_{c}^{-h} q_{1}^{m+\frac{l}{c}}}
\end{aligned}
$$


We only estimate the first summand since the other terms are treated similarly. For this, we write

$$
-\frac{i \zeta_{2 c}^{h} q_{1}^{-\frac{l}{2 c}+\frac{s l}{c}-\frac{3 l^{2}}{2 c^{2}}} e^{\frac{\pi}{12 k z}}}{2\left(q_{1} ; q_{1}\right)_{\infty}\left(1-\zeta_{c}^{h} q_{1}^{-\frac{l}{c}}\right)}=\frac{i}{2} \zeta_{2 c}^{-h} q_{1}^{\frac{l}{2 c}+\frac{s l}{c}-\frac{3 l^{2}}{2 c^{2}}} e^{\frac{\pi}{12 k z}} \sum_{m=0}^{\infty} p(m) q_{1}^{m} \sum_{r=0}^{\infty} \zeta_{c}^{-h r} q_{1}^{\frac{l r}{c}} .
$$

If $s=0$, then the terms contributing to $N^{*}$ are given by

$$
\frac{1}{2} e^{-\frac{\pi l}{2 c}+\frac{3 \pi l^{2}}{2 c^{2}}+\frac{\pi}{24}}\left(\sum_{r \geq r_{0}} e^{-\frac{\pi r l}{c}}+\sum_{r=0}^{\infty} e^{-\frac{\pi r l}{c}} \sum_{m=1}^{\infty} p(m) e^{-\pi m}\right)
$$

with

$$
r_{0}:=\left\lceil-\frac{1}{2}+\frac{3 l}{2 c}+\frac{c}{24 l}\right\rceil .
$$

It is not hard to see that $(2.10)$ can be estimated against

$$
\frac{e^{-\frac{\pi l}{2 c}+\frac{3 \pi l^{2}}{2 c^{2}}+\frac{\pi}{24}-\frac{\pi r_{0} l}{c}}}{2\left(1-e^{-\frac{\pi l}{c}}\right)}+\frac{e^{-\frac{\pi l}{2 c}+\frac{3 \pi l^{2}}{2 c^{2}}+\frac{\pi}{24}} c_{2}}{2\left(1-e^{-\frac{\pi l}{c}}\right)} \leq \frac{1+e^{\pi \delta_{0}} c_{2}}{2\left(1-e^{-\frac{\pi}{c}}\right)} .
$$

In the case $s \neq 0,(2.9)$ can be bounded by

$$
\frac{1}{2} \frac{e^{-\frac{\pi l}{2 c}+\frac{3 \pi l^{2}}{2 c^{2}}-\frac{\pi s l}{c}+\frac{\pi}{24}}}{1-e^{-\frac{\pi l}{c}}}\left(1+c_{2}\right) \leq \frac{1+c_{2}}{2\left(1-e^{-\frac{\pi}{c}}\right)} .
$$

Thus $N^{*}$ can be estimated against

$$
\frac{2+c_{2}\left(1+e^{\pi \delta_{0}}\right)}{2\left(1-e^{-\frac{\pi}{c}}\right)}+\frac{1}{2} e^{\pi \delta_{0}}\left(1+c_{2}\right)\left(e^{-\pi} c_{3}+c_{1}\right)=: A(c),
$$

where

$$
c_{3}:=\sum_{m=2}^{\infty} \frac{e^{-\frac{\pi m}{2}(3 m+1)+3 \pi m}}{1-e^{\pi-\pi m}} .
$$

We hence obtain the bound

$$
T_{2} \leq 16 A(c)\left|\sin \left(\frac{\pi j}{c}\right)\right| n^{\frac{1}{4}} e^{2 \pi} .
$$

2.2.4. Estimation of $\sum_{3}$. We next consider the error coming from $\sum_{3}$. For this, we write $H_{j, c}^{+}(x)+H_{j, c}^{-}(x)=H_{j, c}(x)$, with $H_{j, c}^{ \pm}(x):= \pm \frac{i}{2 \sin \left(\frac{\pi j}{c}\right) \sinh \left(x \pm \frac{\pi i j}{c}\right)}$.

We denote the contribution of these functions to $I_{j, c, k, \nu}(x)$ by $I_{j, c, k, \nu}^{ \pm}(x)$. Proceeding as in the proof of Lemma 3.1 of [5], we obtain

$$
\begin{aligned}
\left|z^{\frac{1}{2}} I_{j, c, k, \nu}^{ \pm}(z)\right| & \leq \frac{k}{2 \pi|z|^{\frac{1}{2}}\left|\sin \left(\frac{\pi \nu}{k}-\frac{\pi}{6 k} \pm \frac{\pi j}{c}\right)\right|} \frac{1}{\left|\sin \left(\frac{\pi j}{c}\right)\right|} \int_{\mathbb{R}} e^{-\frac{3 k}{\pi} \operatorname{Re}\left(\frac{1}{z}\right) t^{2}} d t \\
& =\frac{\sqrt{k}}{2 \sqrt{3}\left(\operatorname{Re}\left(\frac{1}{z}\right)|z|\right)^{\frac{1}{2}}\left|\sin \left(\frac{\pi j}{c}\right)\right|\left|\sin \left(\frac{\pi \nu}{k}-\frac{\pi}{6 k} \pm \frac{\pi j}{c}\right)\right|} \\
& \leq \frac{2^{-\frac{3}{4}}}{\sqrt{3}} n^{\frac{1}{4}} \frac{1}{\left|\sin \left(\frac{\pi j}{c}\right)\right|\left|\sin \left(\frac{\pi \nu}{k}-\frac{\pi}{6 k} \pm \frac{\pi j}{c}\right)\right|} .
\end{aligned}
$$


This yields the inequality

$$
\sum_{3} \leq \frac{2^{\frac{5}{4}} e^{2 \pi} n^{-\frac{1}{4}}}{\sqrt{3}}\left|\sin \left(\frac{\pi j}{c}\right)\right| \sum_{ \pm} \sum_{k \leq N} \sum_{(\bmod k)} \frac{1}{k\left|\sin \left(\frac{\pi \nu}{k}-\frac{\pi}{6 k} \pm \frac{\pi j}{c}\right)\right|} .
$$

We next estimate the sum on $\nu$. We have

$$
\begin{aligned}
& \sum_{ \pm \quad \nu} \sum_{(\bmod k)} \frac{1}{\left|\sin \left(\frac{\pi \nu}{k}-\frac{\pi}{6 k} \pm \frac{\pi j}{c}\right)\right|}=2 \sum_{\nu} \frac{1}{\mid \bmod k)} \frac{1}{\left|\sin \left(\frac{\pi \nu}{k}-\frac{\pi}{6 k}\right)\right|} \\
&=2\left(\sum_{\nu=1}^{\left[\frac{k}{2}\right]} \frac{1}{\left|\sin \left(\frac{\pi \nu}{k}-\frac{\pi}{6 k}\right)\right|}+\sum_{\nu=0}^{\left[\frac{k+1}{2}\right]-1} \frac{1}{\left|\sin \left(\frac{\pi \nu}{k}+\frac{\pi}{6 k}\right)\right|}\right) \\
& \leq \frac{2 k}{\pi}\left(\sum_{\nu=1}^{\left[\frac{k}{2}\right]} \frac{1}{\left(\nu-\frac{1}{6}\right)\left\{1-\frac{1}{6}\left(\frac{\pi}{k}\left(\left[\frac{k}{2}\right]-\frac{1}{6}\right)\right)^{2}\right\}}\right. \\
&\left.+\sum_{\nu=0}^{\left[\frac{k+1}{2}\right]-1} \frac{1}{\left(\nu+\frac{1}{6}\right)\left\{1-\frac{1}{6}\left(\frac{\pi}{k}\left(\left[\frac{k+1}{2}\right]-\frac{5}{6}\right)\right)^{2}\right\}}\right) \leq \frac{4 k \log \left(\frac{k}{2}\right)}{\pi\left(1-\frac{\pi^{2}}{24}\right)}
\end{aligned}
$$

where $\{x\}:=x-[x]$. Combing the above bounds gives the estimate

$$
\sum_{3} \leq \frac{2^{\frac{9}{4}} \cdot 2^{\frac{1}{4}} e^{2 \pi} n^{\frac{1}{4}}\left|\sin \left(\frac{\pi j}{c}\right)\right| \log \left(\frac{n}{4}\right)}{\sqrt{3} \pi\left(1-\frac{\pi^{2}}{24}\right)} .
$$

2.2.5. Symmetrizing paths of integration. We write

$$
\int_{-\vartheta_{h, k}^{\prime}}^{\vartheta_{h, k}^{\prime \prime}}=\int_{-\frac{1}{k N}}^{\frac{1}{k N}}-\int_{-\frac{1}{k N}}^{-\frac{1}{k\left(k+k_{1}\right)}}-\int_{\frac{1}{k\left(k+k_{2}\right)}}^{\frac{1}{k N}}
$$

and estimate the contributions to the error terms from the last two integrals as before (using $\operatorname{Re}(z)=\frac{k}{n}, \operatorname{Re}\left(\frac{1}{z}\right)<k$, and $|z|^{2} \geq \frac{k^{2}}{n^{2}}$ ). Their contribution to $\sum_{1}$ can be estimated against

$$
\frac{8 e^{2 \pi+\frac{\pi}{12}}\left(1+\log \left(\frac{c-1}{2}\right)\right) \cdot n^{\frac{1}{4}}}{c \pi\left(1-\frac{\pi^{2}}{24}\right)}\left|\sin \left(\frac{\pi j}{c}\right)\right| .
$$

We next consider the error that is introduced by symmetrizing $\sum_{2}$, which can be estimated against

$$
8 e^{2 \pi}\left|\sin \left(\frac{\pi j}{c}\right)\right| \sum_{\substack{r, k \\ \delta_{j, c, k, r}>0}} k^{-\frac{1}{2}} e^{2 \pi \delta_{j, c, k, r}} .
$$

Recall that $\delta_{j, c, k, r}>0$ implies that $s \in\{0,3\}$. For $s=0$, the sum on $r$ equals

$$
e^{-\frac{\pi l}{c}+\frac{3 \pi l^{2}}{c^{2}}+\frac{\pi}{12}} \sum_{r \leq r_{0}-1} e^{-\frac{2 \pi l r}{c}}=\frac{e^{-\frac{\pi l}{c}+\frac{3 \pi l^{2}}{c^{2}}+\frac{\pi}{12}}\left(e^{-\frac{2 \pi l}{c} r_{0}}-1\right)}{e^{-\frac{2 \pi l}{c}}-1} \leq \frac{e^{2 \pi \delta_{0}}}{1-e^{-\frac{2 \pi}{c}}} .
$$


The case $s=3$ is treated similarly and yields the same error term. Thus the error introduced by symmetrizing $\sum_{2}$ can be estimated against

$$
16 e^{2 \pi}\left|\sin \left(\frac{\pi j}{c}\right)\right| n^{\frac{1}{4}} \frac{e^{2 \pi \delta_{0}}}{1-e^{-\frac{2 \pi}{c}}} .
$$

2.2.6. Error introduced by integrating along the smaller arc. To finish the evaluation of $\sum_{1}$ and $\sum_{2}$ we have to consider integrals of the form

$$
I_{k, r}:=\int_{-\frac{1}{k N}}^{\frac{1}{k N}} z^{-\frac{1}{2}} \cdot e^{\frac{2 \pi}{k}\left(z\left(n-\frac{1}{24}\right)+\frac{r}{z}\right)} d \Phi .
$$

Substituting $z=\frac{k}{n}-i k \Phi$ gives

$$
I_{k, r}=\frac{1}{k i} \int_{\frac{k}{n}-\frac{i}{N}}^{\frac{k}{n}+\frac{i}{N}} z^{-\frac{1}{2}} \cdot e^{\frac{2 \pi}{k}\left(z\left(n-\frac{1}{24}\right)+\frac{r}{z}\right)} d z .
$$

We denote the circle through $\frac{k}{n} \pm \frac{i}{N}$ and tangent to the imaginary axis at 0 by $\Gamma$. Writing $z=x+i y, \Gamma$ is given by $x^{2}+y^{2}=\alpha x$, with $\alpha:=\frac{k}{n}+\frac{n}{N^{2} k}$. We change the path of integration into the larger arc by Cauchy's Theorem and then estimate the contribution to the error term from the integral along the smaller arc by using the fact that on the smaller arc we have $2>\alpha>\frac{1}{k}, \operatorname{Re}(z) \leq \frac{k}{n}$, and $\operatorname{Re}\left(\frac{1}{z}\right)<k$. Denoting by $\mathcal{A}$ the smaller arc on the circle, we can estimate the contribution against

$$
\frac{2}{k} e^{2 \pi+2 \pi r} \int_{\mathcal{A}}|z|^{-\frac{1}{2}} d z \leq \frac{1}{k} e^{2 \pi+2 \pi r} \alpha^{-\frac{1}{4}}\left|\frac{4}{3}\left(\frac{k}{n}\right)^{\frac{3}{4}}+i \int_{0}^{\frac{k}{n}} x^{-\frac{3}{4}} \frac{\alpha-2 x}{2 \sqrt{\alpha-x}} d x\right| .
$$

It is not hard to see that the function $f(x):=\frac{\alpha-2 x}{2 \sqrt{\alpha-x}}$ on $[0, \alpha]$ obtains its maximum at $x=0$. Thus we have a contribution of at most

$$
\frac{2}{k} e^{2 \pi+2 \pi r} \alpha^{-\frac{1}{4}}\left(\frac{4}{3}\left(\frac{k}{n}\right)^{\frac{3}{4}}+2 \alpha^{\frac{1}{2}}\left(\frac{k}{n}\right)^{\frac{1}{4}}\right) \leq\left(\frac{4}{3}+2^{\frac{5}{4}}\right) \frac{2}{k} e^{2 \pi+2 \pi r} N^{-\frac{1}{2}}
$$

This yields, using (2.7), that the contribution coming from $\sum_{1}$ can be bounded by

$$
\frac{4\left(\frac{4}{3}+2^{\frac{5}{4}}\right)\left|\sin \left(\frac{\pi j}{c}\right)\right|\left(1+\log \left(\frac{c-1}{2}\right)\right) e^{2 \pi+\frac{\pi}{12}} n^{\frac{1}{4}}}{\pi c\left(1-\frac{\pi^{2}}{24}\right)} .
$$

Similarly the contribution coming from $\sum_{2}$ can be estimated against

$$
8\left|\sin \left(\frac{\pi j}{c}\right)\right|\left(\frac{4}{3}+2^{\frac{5}{4}}\right) \frac{e^{2 \pi \delta_{0}+2 \pi}}{1-e^{-\frac{2 \pi}{c}}} .
$$

Having bounded all of the relevant terms, we conclude Theorem 1.2. 


\section{Appendix}

Following the argument given in the proof of Theorem 1.2 to bound the error terms, one can obtain effective bounds for $c=5, c=7$, and $c=9$ beyond which the main term of the asymptotic formula dominates the other terms. Noting that the sign of the main term is determined by $n$ modulo $c$ easily gives Theorem 1.1 for sufficiently large $n$. Since the upper bounds for integers that might not satisfy the inequalities in Theorem 1.1 are relatively small, we obtain the explicit set of exceptions to Theorem 1.1 after a quick computer check (calculations were done here with MAGMA and MAPLE). For $0 \leq a<b \leq \frac{c-1}{2}$ and $d(\bmod c)$ fixed, Tables 1,2 , and 3 contain the set of $n \equiv d(\bmod c)$ which do not satisfy the inequality given in Theorem 1.1 for $c=5, c=7$, and $c=9$, respectively. For brevity we omit the choices of $(a, b, d)$ for which there are no exceptions.

TABLE 1. Exceptions to the inequality for $c=5$.

\begin{tabular}{cc}
\hline$(a, b, d)$ & Exceptions $n$ \\
\hline$(0,2,0)$ & $\{5,10,20,30,50\}$ \\
$(0,1,2)$ & $\{7,27\}$ \\
$(1,2,2)$ & $\{7,27\}$ \\
$(0,1,3)$ & $\{8\}$ \\
$(0,2,3)$ & $\{3,13\}$ \\
\hline
\end{tabular}

TABle 2. Exceptions to the inequality for $c=7$.

\begin{tabular}{cc}
\hline$(a, b, d)$ & Exceptions $n$ \\
\hline$(0,1,0)$ & $\{14,42,56,70,84,126\}$ \\
$(0,2,0)$ & $\{14,42\}$ \\
$(0,3,0)$ & $\{14,42\}$ \\
$(1,2,0)$ & $\{7,21,28,35,49,63,77,91,133\}$ \\
$(1,3,0)$ & $\{7,21,28,35,49,63,77,91,133\}$ \\
$(0,2,2)$ & $\{2,9,23,30,51\}$ \\
$(2,3,2)$ & $\{2,9,23,30,51\}$ \\
$(0,1,3)$ & $\{10,24,52\}$ \\
$(0,3,3)$ & $\{10,24,52\}$ \\
$(1,2,3)$ & $\{10,24,52\}$ \\
$(2,3,3)$ & $\{11,18,39,52\}$ \\
$(0,2,4)$ & $\{11,18,39,39,53\}$ \\
$(1,2,4)$ & $\{13\}$ \\
$(2,3,4)$ & $\{6,20,34\}$ \\
$(0,1,6)$ & $\{6,20,34\}$ \\
$(0,2,6)$ & $\{13\}$ \\
$(0,3,6)$ & \\
$(1,2,6)$ & \\
$(1,3,6)$ &
\end{tabular}


TABLE 3. Exceptions to the inequality for $c=9$.

\begin{tabular}{cc}
\hline$(a, b, d(\bmod 3))$ & Exceptions $n$ \\
\hline$(0,1,0)$ & $\{3,9,12,15,21,27,33,39,45,57,75\}$ \\
$(0,2,0)$ & $\{3,6,12,15,18,24\}$ \\
$(0,3,0)$ & $\{6\}$ \\
$(0,4,0)$ & $\{3,12,15,21,39\}$ \\
$(1,2,0)$ & $\{3\}$ \\
$(1,3,0)$ & $\{3\}$ \\
$(1,4,0)$ & $\{6,9\}$ \\
$(2,3,0)$ & $\{4\}$ \\
$(2,4,0)$ & $\{4,16\}$ \\
$(0,1,1)$ & $\{1,7,13,19,25\}$ \\
$(0,3,1)$ & $\{1,4,10\}$ \\
$(1,2,1)$ & $\{1,7\}$ \\
$(1,3,1)$ & $\{1,7\}$ \\
$(1,4,1)$ & $\{1,4,10\}$ \\
$(2,3,1)$ & $\{1\}$ \\
$(2,4,1)$ & $\{5\}$ \\
$(3,4,1)$ & $\{2,5,14\}$ \\
$(0,1,2)$ & $\{2,8\}$ \\
$(0,2,2)$ & $\{5\}$ \\
$(0,3,2)$ & $\{2\}$ \\
$(1,2,2)$ & $\{2,5,14\}$ \\
$(1,4,2)$ & $\{2,8\}$ \\
$(2,3,2)$ & \\
$(2,4,2)$ & \\
$(3,4,2)$ &
\end{tabular}

\section{Acknowledgements}

The authors thank J. Lovejoy and K. Mahlburg for fruitful conversations.

\section{References}

[1] G. Andrews, On the theorems of Watson and Dragonette for Ramanujan's mock theta functions, Amer. J. Math. 88 (1966), 454-490.

[2] G. Andrews and F. Garvan, Ramanujan's "lost" notebook. VI. The mock theta conjectures, Adv. in Math. 73 (1989), 242-255.

[3] G. Andrews and R. Lewis, The ranks and cranks of partitions moduli 2, 3, and 4, J. Number Theory 85 (2000), 74-84.

[4] A. Atkin and H. Swinnerton-Dyer, Some properties of partitions, Proc. London Math. Soc. 4 (1954), 84-106.

[5] K. Bringmann, Asymptotics for rank partition functions, appeared: Trans. Amer. Math. Soc. 361 (2009), pages 3483-3500.

[6] K. Bringmann and K. Ono, The $f(q)$ mock theta function conjecture and partition ranks, Inv. Math. 165 (2006), 243-266.

[7] K. Bringmann and K. Ono, Dyson's ranks and Maass forms, appeared: Ann. of math. 171 (2010), pages 419-449.

[8] K. Bringmann and K. Ono, Coefficients of harmonic weak Maass forms, accepted for publication, Proceedings of the 2008 University of Florida Conference on Partitions, q-series, and modular forms. 
[9] K. Bringmann, K. Ono, and R. Rhoades, Eulerian series as modular forms, J. Amer. Math. Soc. 21 (2008), 1085-1104.

[10] L. Dragonette, Some asymptotic formulae for the mock theta series of Ramanujan, Trans. Amer. Math. Soc. 72 (1952), 474-500.

[11] F. Dyson, Some guesses in the theory of partitions, Eureka (Cambridge) 8 (1944), 10-15.

[12] F. Garvan, Combinatorial interpretations of Ramanujan's partition congruences, Ramanujan revisited (Urbana-Champaign, Ill., 1987), 29-45, Academic Press, Boston, MA, 1988.

[13] F. Garvan, New combinatorial interpretations of Ramanujan's partition congruences mod 5, 7 and 11, Trans. Amer. Math. Soc. 305 (1988), 47-77.

[14] D. Hickerson, A proof of the mock theta conjectures, Invent. Math. 94 (1988), 639-660.

[15] S. Kang, Mock Jacobi forms in basic hypergeometric series, preprint.

[16] M. Knopp, W. Kohnen, W. Pribitkin, On the signs of Fourier coefficients of cusp forms, Ramanujan Journal 7 (2003), 269-277.

[17] R. P. Lewis, The ranks of partitions modulo 2, Discuss. Math. 167/168 (1997), 445-449.

[18] H. Rademacher, Topics in analytic number theory, Springer-Verlag, New York-Heidelberg, 1973.

[19] S. Ramanujan, The lost notebook and other unpublished papers, Narosa, New Delhi, 1988.

[20] N. Santa-Gadea, On some relations for the rank moduli 9 and 12, J. Number Theory 40 (1992), 130-145

[21] S. Zwegers, Mock theta functions, Ph.D. Thesis, Universiteit Utrecht, 2002.

[22] S. Zwegers, Mock $\vartheta$-functions and real analytic modular forms, $q$-series with applications to combinatorics, number theory, and physics (Ed. B. C. Berndt and K. Ono), Contemp. Math. 291, Amer. Math. Soc., (2001), pages 269-277.

Mathematisches Institut, Universität Köln, Weyertal 86-90, D-50931 Köln, Germany

E-mail address: kbringma@math.uni-koeln.de

Wiskunde Afdeling, Radboud Universiteit, Postbus 9010, 6500 GL, Nijmegen, NetherLANDS

E-mail address: bkane@science.ru.nl 